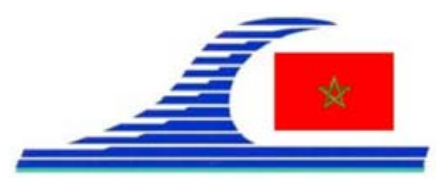

Conférence Méditerranéenne Côtière et Maritime EDITION 2, TANGER, MAROC (2011)

Coastal and Maritime Mediterranean Conference

Disponible en ligne - http://www.paralia.fr - Available online

\title{
Ensablement et pollution par les métaux lourds des sédiments du port de Ténès
}

\section{Rabah BELKESSA $^{1}$, Samir BACHOUCHE ${ }^{1}$, Fouzia BACHARI HOUMA ${ }^{1}$}

1. Ecole Nationale Supérieure des Sciences de la Mer et de l’Aménagement du Littoral.

Campus Universitaire de Dely Ibrahim Bois des Cars, 16320 Alger, Algérie.

belkessarabah@yahoo.fr ; bachouche.samir@gmail.com ;

bacharifouzia.ocean@gmail.com

\section{Résumé :}

Comme tous les ports algériens, le port de Ténès souffre d'un ensablement accru, par sa situation géographique (compris entre deux grands cours d'eau), il est soumis à des apports importants de sédiments d'autant plus qu'il possède deux passes d'entrée. Les activités économiques du port, les travaux de carénage des bateaux ainsi que les différents rejets effectués au niveau port introduisent des polluants au niveau des sédiments du port. Cette pollution des sédiments pourrait poser un problème quant au devenir des produits de dragage (METAP, 1994).

Les ports qui sont des milieux fermés, favorisent la sédimentation des sédiments et en particulier la fraction fine qui constitue un piège pour les métaux lourds.

Le but de ce travail est de connaitre d'une part, la granulométrie des sédiments du port et d'autre part connaitre la concentration de quelques métaux lourds.

Mots clés :

Ensablement - Port - Sédiment - Pollution - Dragage

\section{Introduction}

Le port de Ténès est ancré dans la petite baie de Ténès entre cap Kalah et cap Ténès, il est situé à $240 \mathrm{~km}$ à l'Ouest d'Alger et à $160 \mathrm{~km}$ de Mostaganem et a comme coordonnées $36^{\circ} 32^{\prime}$ de latitude Nord et $01^{\circ} 32^{\prime}$ de longitude Est.

Le port est formé par deux jetées (Est de $400 \mathrm{ml}$ et Ouest de $700 \mathrm{ml}$ ) séparées par une ouverture de $160 \mathrm{~m}$, et protégé par une brise lame de $450 \mathrm{~m}$.

La surface du plan d'eau est de 20 ha avec des profondeurs de $12 \mathrm{~m}$ à la passe Est et $14 \mathrm{~m}$ à la passe Ouest. Les activités sont commerciales de pêche et de plaisance.

L'oued Alala qui débouche près de la ville de Ténès transporte des quantités de sédiments assez importantes.

\section{Evolution bathymétrique du port de Ténès}

Les comparaisons faites sur plusieurs années montrent les levés bathymétriques sont très différents 
La connaissance de la Mer :

un vecteur du développement durable en Méditerranée

De 1961, 1974 de 1982, 1984, 1995 et celui de 2000 nous ont permis de comprendre les échanges sédimentologiques entre l'extérieur et l'intérieur du port (figure 1).

Entre 1961 et 1974, la profondeur a augmenté de 3,52 m. Il est à noter qu'un dragage a eu lieu en 1973 au niveau de la passe. La différence bathymétrique entre 1974 et 1982 est en moyenne de $-1,32 \mathrm{~m}$ soit un ensablement de $16 \mathrm{~cm} / \mathrm{an}$. Il semble que l'ensablement soit vraiment net entre $1982(7,38 \mathrm{~m})$ et $1984(4,18 \mathrm{~m})$ soit une différence moyenne de $-3,20 \mathrm{~m}(1,6 \mathrm{~m} / \mathrm{an})$. L'exhaussement des fonds entre $1984(-4,18 \mathrm{~m})$ et 1995 (-6,2 $0 \mathrm{~m})$ est de 2,02 $\mathrm{m}$ soit un départ de sédiment de 2,02 m (18 cm/an). La différence bathymétrique moyenne entre 1995 (6,20 m) et 2000 (8,25 m) est de 2,02 m soit un exhaussement de fond 0,40 m/an. En comparant toutes les données bathymétriques moyennes entre 1961 et 2000, l'ensablement est estimé à 0,15 m/an soit une épaisseur de 5,85 m.

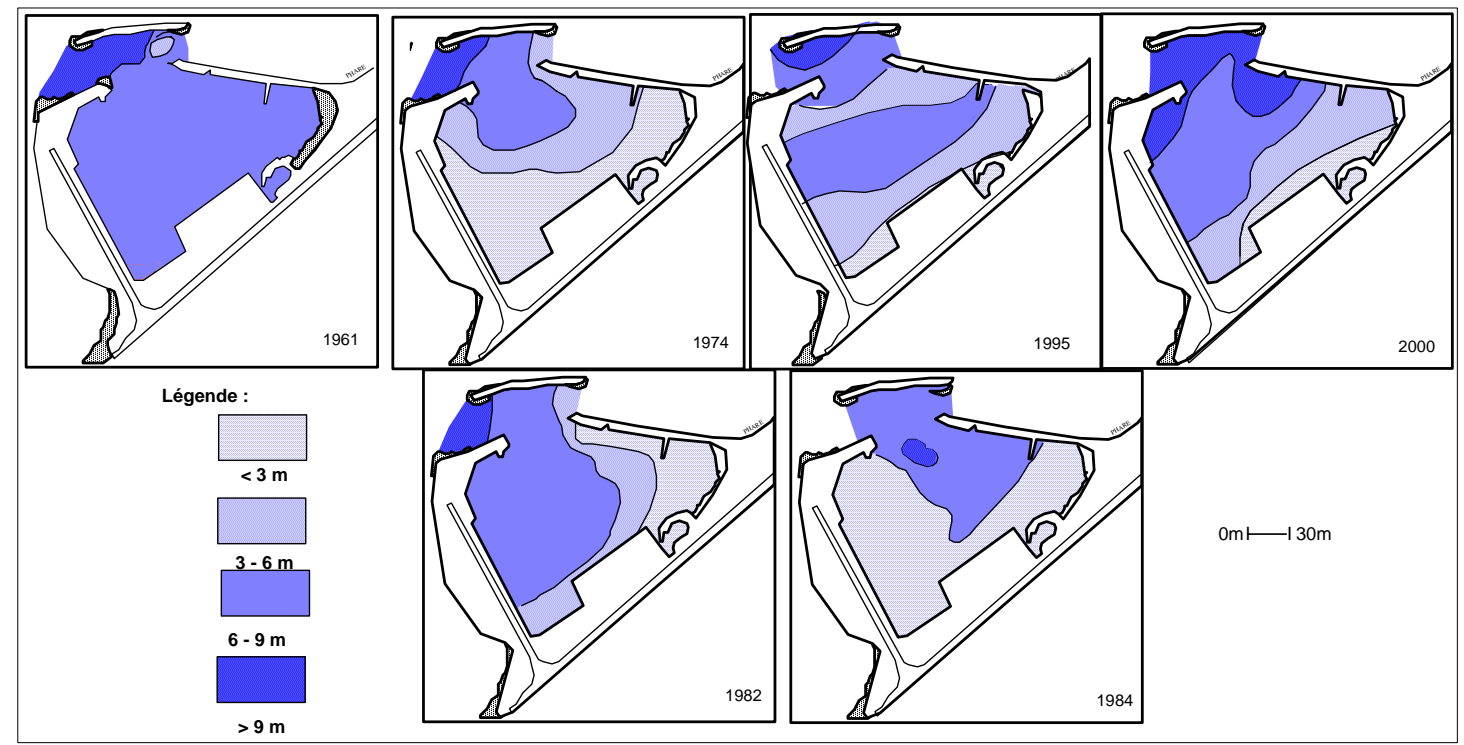

Figure 1. Evolution diachronique des fonds du port de Ténès.

\section{Granulométrie dans le port de Ténès}

Les sédiments qui arrivent dans le port suivent un granoclassement au fur et à mesure qu'on pénètre à l'intérieur du port. La concentration la plus élevée en pélites est rencontrée au milieu du port (> à 50\%). Les plus faibles taux sont retrouvés entre le brise lame et la passe d'entrée (< à 5\%). Les abords quais sont tapissés par de pélites dont les pourcentages se situent entre $5 \%$ et $50 \%$. Cette configuration dénote un déplacement circulatoire des sédiments dans le bassin avec une zone calme au niveau du port (figure 2).

On distingue 3 grandes populations de sables dans le port (BERTHOIS, 1975) (figure 5). Des sables très fins $(<125 \mu \mathrm{m})$ au niveau de la passe d'entrée, des sables fins 
(125 $\mu \mathrm{m}$ - $250 \mu \mathrm{m})$ qui décrivent sur un petit espace une entrée de sédiments de part et d'autre du brise lame. Les sables grossiers (> $550 \mu \mathrm{m}$ ) se localisent le long des quais. La configuration générale de la distribution de la médiane dénote l'entrée des sédiments par les 2 passes d'entrée. La répartition du Q25 montre un transit comparable du Q25 que celui des médianes et des pélites. Les sables grossiers se situent au niveau de la passe Est et pénètrent jusqu'au fond du port. Les sables moyens longent la jetée Ouest, entre ces 2 fasciés c'est le domaine des sables très fins (figure 4).

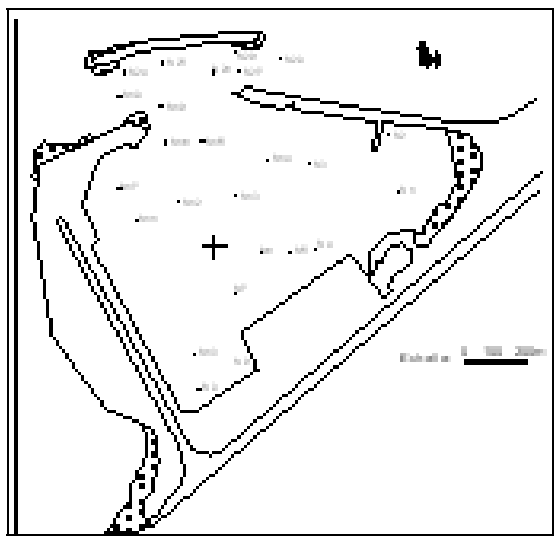

Figure 2a. Situation des stations de prélèvement.

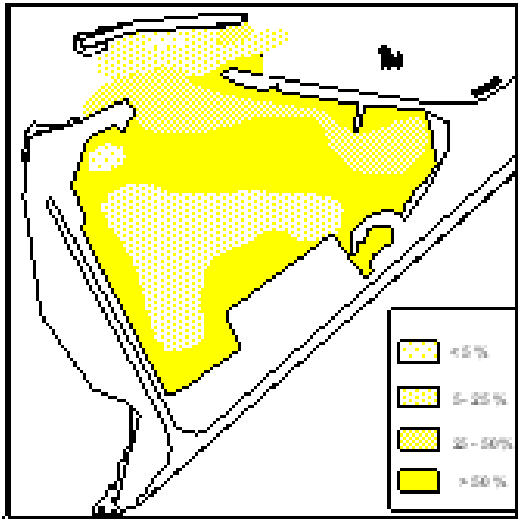

Figure 2b. Répartition des pélites.

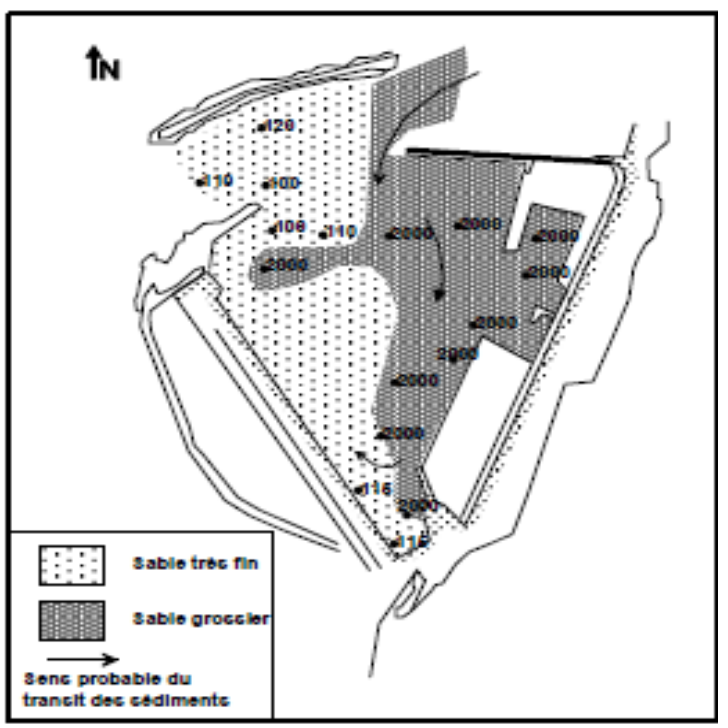

Figure 3. Répartition du Q75.

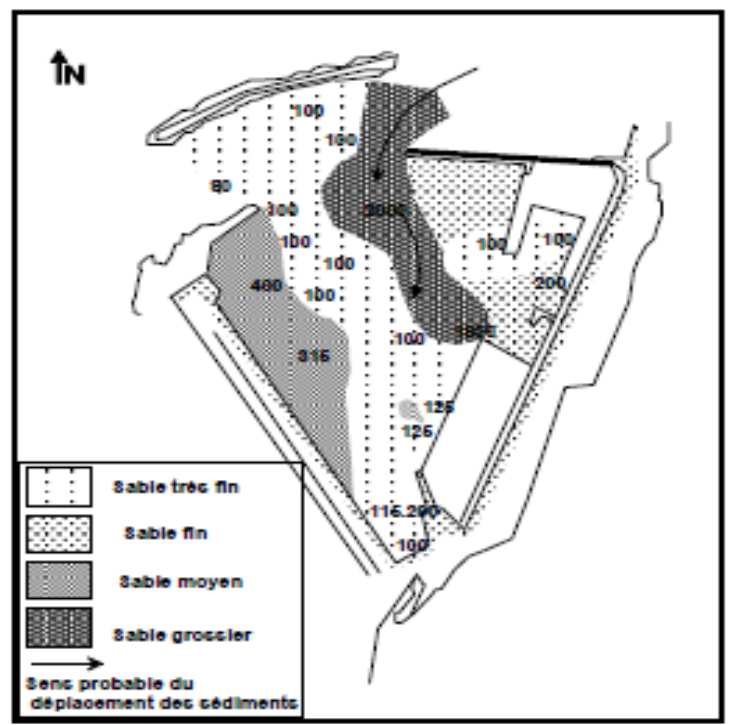

Figure 4. Répartition du Q25. 
La connaissance de la Mer :

un vecteur du développement durable en Méditerranée

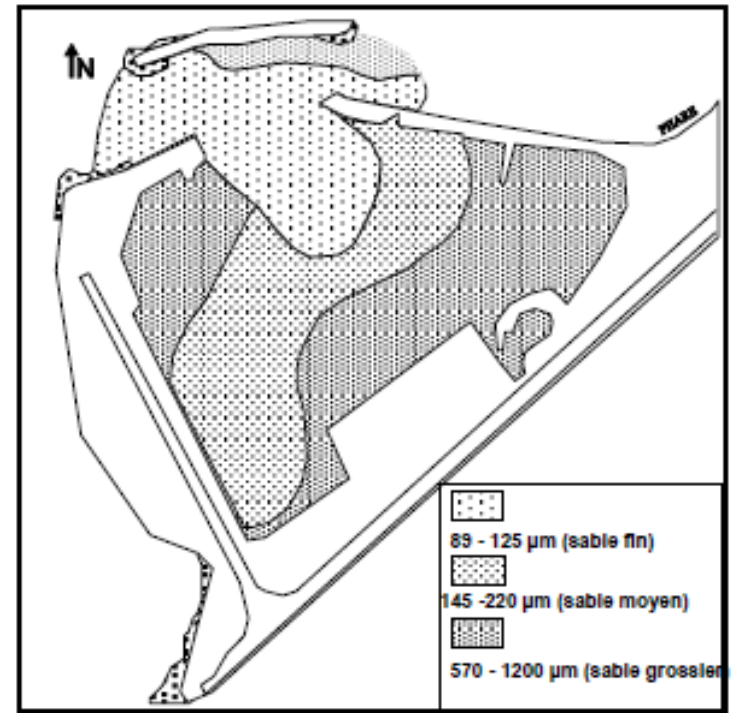

Figure 5. Répartition du Q50.

Le long de la jetée Est et de la darse de pêche on retrouve les sables fins. La répartition de Q25 témoigne d'un transfert de sédiments à partir de la passe Est qui longeraient les quais pour s'arrêter au niveau de la jetée Ouest. Le centre du port (sable très fin) traduit une zone calme. La répartition du Q75 montre une entrée des sédiments par la passe Est pour occuper les 3/4 Est du port. La partie Ouest est contrairement à celle de l'Est parsemée de sables très fins (figure 3).

Afin de comprendre la circulation probable des sédiments sur le fond du port, deux flotteurs de $30 \mathrm{~cm}$, lestés de $2 \mathrm{~kg}$ à $1,5 \mathrm{~m}$ de profondeur de diamètre et lestés ont été largués prés de la passe d'entrée Est, leur suivi a été effectué à l'aide de théodolites et positionné toutes les 10 minutes. Par une houle et un vent Nord Est, les flotteurs ont contourné la digue Est. Le premier flotteur s'est arrêté au début de la digue Ouest, le second a pris la même trajectoire que le premier jusqu'au milieu du bassin pour s'arrêter. Pour une houle de direction Nord, où le brise lame offre un obstacle important, les flotteurs suivent le même sens que lors des premiers essais. Leur stationnement s'est effectué au milieu du port.

\section{Pollution par les métaux lourds dans les sédiments du port de Ténès}

Les échantillons choisis pour l'analyse des métaux lourds (par SAA) ont été prélevés dans les sédiments (CHARLOU \& JOANNY, 1983). Cinq stations ont été étudiées, trois à l'intérieur du port et deux à l'extérieur.

Les concentrations en mercure montrent des valeurs qui se situent en 0,01 $\mu \mathrm{g} / \mathrm{g}$ (station 4 à l'extérieur du port) et 0,25 $\mu \mathrm{g} / \mathrm{g}$ (station 2). Les sédiments présentent une pollution identique aussi bien en surface qu'en profondeur (à $20 \mathrm{~cm}$ du fond). 
Pour les normes algériennes, les valeurs sont en dessous des normes, pour les normes françaises $(0,20 \mu \mathrm{g} / \mathrm{g})$, la station 2 présente une pollution en mercure.

Les concentrations en cadmium montrent des valeurs se situant entre $0,15 \mu \mathrm{g} / \mathrm{g}$ (station 3) et 1,25 $\mu \mathrm{g} / \mathrm{g}$ (station 2) et sont au-dessus des normes françaises.

Les concentrations en plomb varient entre $23 \mu \mathrm{g} / \mathrm{g}$ (station 5) et $125 \mu \mathrm{g} / \mathrm{g}$ (station 2).Toutes les stations présentent une pollution par le plomb pour les normes françaises.

Les valeurs en cuivre sont presque homogènes dans toutes les stations, avec des concentrations légèrement élevées pour la station 1 et 2 . Pour les normes françaises les 3 stations du port sont au dessus du seuil toléré.

Les concentrations en zinc pour la station 2, présentent des valeurs très élevées (380 $\mu \mathrm{g} / \mathrm{g}$, station 2), la situant au-dessus des normes françaises. Les autres stations présentent des valeurs presque identiques. La station $1(155 \mu \mathrm{g} / \mathrm{g})$ est au-dessus des normes européennes.

Les valeurs des indices de contamination pour les normes nationales montrent des taux de pollution assez faibles dans le cas des métaux lourds. Seule la station 2 et pour les cas du plomb et du zinc présente un petit risque de pollution (IC entre 3 et 10).

\section{Dragage du port de Ténès}

Les données des côtes à draguer ont été fournies par le Ministère des Travaux Publics (D.I.M) et se répartissent comme suit :

La passe Est et Ouest (-12 m), la passe principale (- $8 \mathrm{~m})$, quai de pêche (- 4,5 m), quai commercial (-5 m).

Estimation des volumes des déblais : Les secteurs de dragage ont été répartis en huit zones. Par rapport à la bathymétrie actuelle, seules les zones intérieures du port (3,5 m, $2 \mathrm{~m}$ et $5 \mathrm{~m}$ ) ont une épaisseur de sédiments à draguer élevée. Dans les autres zones, les déblais se répartissent sur une épaisseur entre 0,5 m et $1 \mathrm{~m}$. (BELKESSA, 2004).

Le total des sédiments à draguer dans le port de Ténès (2009) est estimé à $246100 \mathrm{~m}^{3}$.

\section{Références Bibliographiques}

BELKESSA R. (2004). Reconnaissance de sites de dépôts des produits de dragage portuaires sur le plateau et le littoral algériens (méthodologie et paramètres d'étude). contrat Ismal/Cnepru.

BERTHOIS L (1975). Etude sédimentologique des roches meubles. Doin Editeur, Paris, $278 \mathrm{p}$.

CHARLOU J., JOANNY M. (1983). Dosage du mercure et d'autres métaux ( $\mathrm{Pb}, \mathrm{Zn}$, $\mathrm{Cu}, \mathrm{Cd}, \mathrm{Co}, \mathrm{Ni}, \mathrm{Cr}, \mathrm{Mn}$ ) dans les sédiments marins par absorption atomique. In Aminiot A \& Chaussepied M. (Eds) : Manuel des analyses chimiques en milieu marin. Bndo/Document, Brest, pp 285-295. 
La connaissance de la Mer :

un vecteur du développement durable en Méditerranée

METAP -Ministère des transports, Direction des Ports Algérie- (1994). Etude de protection contre la pollution des ports et du littoral algérien. Banque européenne d'investissement, Rapport final (juin 1994), 170 p. 